\title{
Dynamics of one-dimensional Bose liquids: Andreev-like reflection at Y-junctions and absence of the Aharonov-Bohm effect
}

\author{
Akiyuki Tokuno ${ }^{1,2}$, Masaki Oshikawa ${ }^{1}$, Eugene Demler ${ }^{3}$ \\ ${ }^{1}$ Institute for Solid State Physics, University of Tokyo, Kashiwa 227-8581 Japan \\ 2 Department of Physics, Tokyo Institute of Technology, Oh-okayama, Meguro-ku, Tokyo 152-8551 Japan \\ 3 Department of Physics, Harvard University, MA 02138
}

(Dated: September 23, 2018)

\begin{abstract}
We study one dimensional Bose liquids of interacting ultracold atoms in the Y-shaped potential when each branch is filled with atoms. We find that the excitation packet incident on a single Y-junction should experience a negative density reflection analogous to the Andreev reflection at normal-superconductor interfaces, although the present system does not contain fermions. In a ring interferometer type configuration, we find that the transport is completely insensitive to the (effective) flux contained in the ring, in contrast to the Aharonov-Bohm effect of a single particle in the same geometry.
\end{abstract}

PACS numbers: 03.75.Kk

Recently, guiding of atoms in low-dimensionally magnetic traps has been actively studied [1]. Such systems provide an ideal opportunity to study coherent quantum dynamics of interacting many-body systems. The theory of one-dimensionally trapped atoms in equilibrium [2, 3, 4, 5], as well as questions related to coherent dynamics and nonequilibrium phenomena [ $[$ ], has been vigorously studied. In this Letter we analyze a simple yet nontrivial example of the real-time dynamics of BoseEinstein condensates (BEC) in the Y-shaped potentials (beam-splitters) 7], and in the related "ring interferometer" type geometry [8].

In contrast to previous works where the dynamics of wave packets in otherwise empty traps is studied, here we consider the situation where each one-dimensional branch of the Y-shaped potential is filled with interacting bosonic atoms, i.e. each branch contains one-dimensional Bose liquid. The Tomonaga-Luttinger (TL) liquid theory [3, 9] is a powerful method to study this problem. The whole system in the Y-shaped potential may be regarded as three TL liquids connected at a junction, as in Fig. 1. While there are parallels with the corresponding electron problem [10, 11], as we will show in the following, a quite different generic behavior is expected in the Bose liquid. In the present case, instead of the conductance, the real-time dynamics could be directly observed in experiments. Furthermore, as an application of the above analysis of the single Y-junction problem, we also consider the transport in a ring type interferometer geometry.

We have two main results in this Letter. The first is the prediction of the negative density reflection at a junction (Fig. 1). In the discussion of $\mathrm{Y}$ junctions for systems of interacting electrons, such negative reflection has been interpreted as the Andreev reflection, which occurs in metal-superconductor interfaces due to Cooper pair formation. On the other hand, the negative reflection at the BEC Y-junction can be understood rather simply using an analogy with electromagnetic transmission line three-way junction. For an ideal three-way junction, there should be no voltage difference between the connected ends of the three lines, and there is conservation of the total charge in a pulse. These two conditions imply that when the amplitude of an incident signal is one, signals transmitted into the other two lines will have amplitudes of $2 / 3$ and the reflected signal will have the amplitude of $-1 / 3$. As we discuss below the same phenomenon should appear in a BEC Y-junction.

The other main result is the absence of the AharonovBohm (AB) effect in the ring type interferometer, which consists of two Y-shaped junctions as in Fig. 2. As a typical example of the $\mathrm{AB}$ effect, when the ring is empty, a single particle injected from one lead to the ring does not transmit to the other lead when the (effective) "magnetic flux" inside the ring is the half unit flux quantum. However, we find that the transport between the leads in our system is completely insensitive to the magnetic flux, in the low energy limit. These two findings are manifestation of the collective nature of the Bose liquid, rather than single-particle physics.

We start from the following one-dimensional Hamiltonian [12] with the low-energy scattering characterized by the low-energy s-wave scattering:

$$
\mathcal{H}_{0}=\sum_{j=1}^{3} \int d x\left[\frac{1}{2 m} \partial_{x} \psi_{j}^{\dagger}(x) \partial_{x} \psi_{j}(x)+\frac{U}{2} \rho_{j}^{2}(x)\right],
$$

where we take an unit of $\hbar=1 . \quad \psi_{j}(x), \rho_{j}(x) \equiv$ $\psi_{j}^{\dagger}(x) \psi_{j}(x)$ are, respectively, the annihilation and the density operator of the boson with the mass $m$, on the $j$-th branch. $(j=1,2,3$. $)$ The effective interaction strength $U>0$ is determined by the s-wave scattering length. We take $x>0$, so the physics at the junction is represented by the boundary condition (b.c.) at $x=0$. In case of the junction problem, the b.c. is a non-trivial problem and plays a crucial role. First of all, we have 
to identify what kind of b.c. describes the physics in the low-energy limit.

Any b.c. should satisfy the total current conservation

$$
J_{1}(t, x=0)+J_{2}(t, x=0)+J_{3}(t, x=0)=0
$$

where $J_{j}(x)$ is the current operator of the Bose liquid in the $j$-th branch. The simplest possible b.c. corresponds to an infinitely strong barrier at the junction, which prohibits any particle to be transmitted through the junction. It would give $J_{j}(t, x=0)=0$ for $j=1,2,3$. Naturally, this satisfies the current conservation (2).

In practice, there would be a finite tunneling amplitude $\Gamma$ between the different branches. The effect of the tunneling can be included by adding the perturbation

$$
\mathcal{H}_{B}=-\sum_{j=1}^{3}\left[\Gamma \psi_{j}^{\dagger}(x=0) \psi_{j-1}(x=0)+\text { h.c. }\right],
$$

at the boundary. We are interested in the low-energy behavior of the junction in the presence of the tunneling.

In order to study the low-energy physics, it is convenient to use the "bosonization" technique [9]. In terms of the TL boson field $\theta_{j}(x)$ and its dual $\varphi_{j}(x)$, the Bose atom annihilation operator and density operator on the $j$-th branch are, respectively, represented as $\psi_{j}^{\dagger}(x) \sim$ $\left[\rho^{(0)}+\partial_{x} \theta_{j}(x) / \pi\right]^{1 / 2} e^{-i \varphi_{j}(x)}, \rho_{j}(x) \sim \rho^{(0)}+\partial_{x} \theta_{j}(x) / \pi$, where we have retained only the leading terms. $\rho^{(0)}$ represents the expectation value of the atom density in the ground state. The low-energy effective Hamiltonian for equation (11) is given by

$$
\mathcal{H}_{0}=\sum_{j=1}^{3} \frac{v}{2 \pi} \int_{0}^{L} d x\left[g\left(\partial_{x} \varphi_{j}\right)^{2}+g^{-1}\left(\partial_{x} \theta_{j}\right)^{2}\right] .
$$

The fields $\theta, \varphi$ satisfy the commutation relation $[\theta(x), \varphi(y)]=i \pi H(x-y)$, where $H(x-y)$ is the Heaviside step function. $v$ is the velocity of the collective modes, and $g$ is the so-called TL parameter, in which the interaction is essentially contained.

The TL parameter, for the interacting fermions i.e. electrons, usually satisfies $g<1$ due to the Coulomb repulsion. However, $g>1$ occurs rather naturally in a Bose liquid with a short-range repulsive interaction. For example, for the Bose liquid with a $\delta$-function interaction [13], the TL parameter $g$ can be determined from the Bethe Ansatz exact solution. It turns out that $g$ is always larger than 1 for this model, interpolating between $g=\infty$ in the non-interacting limit and $g=1$ in the strongly interacting (or dilute) "Tonks gas" limit. [3, 4, 5] While $g$ can also be less than 1 with other types of the interaction [14], $g>1$ would be rather generic for the interacting Bose liquid.

In terms of the bosonization, the "disconnected" limit $\psi_{j}(0)=0$ corresponds to the Dirichlet boundary condition $\partial_{t} \theta_{j}(0)=0$ for $j=1,2,3$. It is equivalent to the
Neumann b.c. on $\varphi_{j}$. The tunneling perturbation (3) is bosonized as $\mathcal{H}_{B} \sim \Gamma e^{-i\left(\varphi_{j}(0)-\varphi_{j-1}(0)\right)}$, where only the most relevant term is retained. The scaling dimension of this perturbation is given by $g^{-1}$, and it is relevant when $g>1$. Namely, the effective tunneling amplitude $\Gamma$ grows as the energy scale such as the temperature is lowered. It is expected that $\Gamma$ eventually grows to infinity in the low-energy limit, when $g>1$. [15]

In the strong coupling limit, the $\varphi$ fields should be pinned as $\varphi_{1}(t, 0)=\varphi_{2}(t, 0)=\varphi_{3}(t, 0)$, because the tunneling term acts as an infinitely strong potential on $\varphi$ at the junction. This also implies $\partial_{t} \varphi_{1}=\partial_{t} \varphi_{2}=\partial_{t} \varphi_{3}$. Translating it into the b.c.s on $\theta_{j}$, we find

$$
\begin{aligned}
& \sum_{j=1}^{3} \partial_{t} \theta_{j}(t, x=0)=0, \\
& \partial_{x} \theta_{i}(t, x=0)=\partial_{x} \theta_{j}(t, x=0)(i, j=1,2,3),
\end{aligned}
$$

where the first line comes from the current conservation (2). The second line indicates that the density is continuous at the junction, reflecting that the junction is at the strong coupling limit. Using the boundary conformal field theory techniques, we can see that all the possible perturbations to the above b.c. are irrelevant if $g>3 / 4[10,11]$ in the case of Bose liquid. This guarantees the stability of the "strong coupling" fixed point, supporting that $\Gamma$ grows to infinity in the low-energy limit when $g>1$.

Now we discuss the dynamics of the system with the stable, strongly coupled b.c.. Let us suppose that the density is altered locally from the equilibrium. The variation of the density propagates in the system, and eventually would be transmitted to other branches.

We start from the Heisenberg equation of motion for the TL boson field $\theta_{j}$ and dual field $\varphi_{j}(j=1,2,3)$ : $\partial_{t} \theta_{j}(t, x)=-v g \partial_{x} \varphi_{j}(t, x), \partial_{t} \varphi_{j}(t, x)=-\frac{v}{g} \partial_{x} \theta_{j}(t, x)$. Let us focus on the expectation values of the density and current. Instead of operators $\theta_{j}$ and $\varphi_{j}$, we then just need to determine the expectation values $\left\langle\theta_{j}\right\rangle$ and $\left\langle\varphi_{j}\right\rangle$, which obey the simple one-dimensional classical wave equation. The initial conditions are given as the expectation values of current and density at $t=0$.

For simplicity, we consider the situation where a leftmoving packet is injected from one side (branch 1 ). Since the left movers go towards the boundary $(x=0)$, and the right movers go away from the boundary, the initial conditions may be given as $\left\langle\rho_{j}^{L}(t=0, x)\right\rangle=\rho^{(0)}+\mathcal{D}_{0}(x) \delta_{1, j}$, $\left\langle\rho_{j}^{R}(t=0, x)\right\rangle=\rho^{(0)}$, where $\mathcal{D}_{0} \equiv \partial_{x}\left\langle\theta_{1}^{L}(t=0, x)\right\rangle / \pi$ is the scalar which gives the initial condition on the expectation value of the densities. Solving the classical wave equation with the initial conditions, we obtain:

$$
\begin{aligned}
\left\langle\rho_{1}(t, x)\right\rangle & =\rho^{(0)}+\mathcal{D}_{0}\left(\eta^{+}\right)-\frac{1}{3} \mathcal{D}_{0}\left(\eta^{-}\right) H\left(\eta^{-}\right), \\
\left\langle\rho_{2,3}(t, x)\right\rangle & =\rho^{(0)}+\frac{2}{3} \mathcal{D}_{0}\left(\eta^{-}\right) H\left(\eta^{-}\right),
\end{aligned}
$$



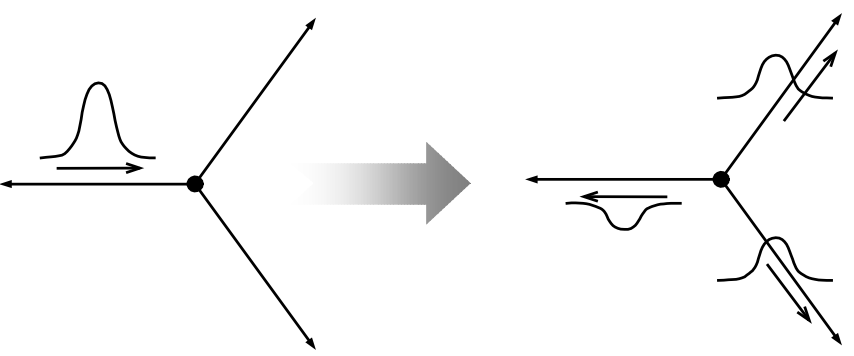

FIG. 1: Reflection and the transmission of the injected current. The left panel describes the initial situation, and the right panel shows the situation after the reflection and the transmission.

where $\eta^{ \pm} \equiv v t \pm x$. The second term in the rhs of the equation (7) represents the incident packet density, and the third term implies the (negative) reflection at the boundary. Namely, if a bump in the density moves through the branch 1 to the junction, a dip in the density is reflected back. (see Fig 1)

Eq. (8) shows the transmission density from the branch 1. Defining the transmission tensor as the ratio of the incident current and transmission currents, the transmission tensor from the branch 1 to the branch 2 (branch 3), $T_{2,1}\left(T_{3,1}\right)$, are obtained as $T_{2,1}=T_{3,1}=\frac{2}{3}$. This means that the sum of the densities transmitted to branches 2 and 3 is greater than the incident density from the branch 1. The current conservation law is satisfied with the "negative reflection" current in the branch 1 , which appears as the second term in equation (77). The time evolution of the density (7)-(8), including the remarkable negative reflection, could be observed in experiments, with present techniques on atomic BEC.

A similar behavior was found in the Y-junction of onedimensional electron systems, in terms of the conductance. It was argued as a consequence of Andreev reflection similar to that in a superconductor-normal interface. What is surprising here is that the Andreev-like negative reflection is also found for the boson systems. The system of bosons is usually not related to Cooper pair formation, which is the underlying mechanism of the Andreev reflection. These results may be also derived from a linearized mean-field theory which is applicable to the weakly interacting regime. While the mean-field approximation breaks down in the strongly interacting regime, our derivation based on the TL liquid theory are valid even in the presence of strong fluctuation. Thus our results represent universal feature of general repulsively interacting boson systems.

Let us extend our analysis to a "ring interferometer" type configuration, which consists of two Y-junctions. (see Fig 2) We take the TL fields on the upper and lower arc in the ring to $\theta_{u, l}$, respectively. Then the symmetric combination $\theta_{s}=\theta_{u}+\theta_{l}$ is coupled with the right and left leads, while anti-symmetric combination $\theta_{a}=\theta_{u}-\theta_{l}$ is

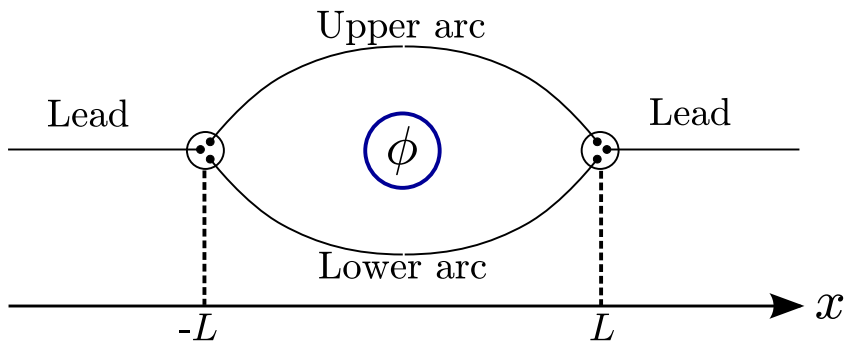

FIG. 2: The schematic figure of the ring interferometer made of two Y junctions, with left and right leads, where $\phi$ is an effective "magnetic" flux enclosed by the ring.

decoupled. Here, the propagation of the atoms in the ring are symmetric so that anti-symmetric combination does not affect the propagation. As a result, we can obtain the inhomogeneous TL liquid [16] as the effective model of the double $\mathrm{Y}$ junction systems, where the TL parameter on the left and right lead is $g$, and that of the symmetric combination $\theta_{s}$ is $2 g$. Following Ref. [17], we can investigate the transmission and the reflection at the junctions. The solution of the equation of motion naturally gives the contribution of multiple reflections. Interestingly, the effect of normal and Andreev-type reflections cancel out, so that the total transmission approaches 1 as the elapsed time approaches infinity. Experimentally, real-time observations of the density could resolve each reflection.

Now let us consider the effect of a "magnetic flux" $\phi$ inside the ring. The present geometry is a typical setting to observe the $\mathrm{AB}$ effect. If we consider the transport of a single charged particle in the same geometry with an empty ring, the transmission probability from the left lead to the right vanishes when $\phi$ is the half unit flux quantum. This is because of the cancellation between the probability amplitudes coming from the two paths. Although a magnetic flux actually does not induce the $\mathrm{AB}$ effect for neutral atoms, several possible approaches for introducing gauge fields and $\mathrm{AB}$ type phase factors for neutral atoms have already been discussed in the literature [18]. A common ingredient of many methods is that of the Berry's phase arising from the orbital motion of atoms [19].

We find that, however, in our problem of filled onedimensional BEC, the transport between the leads is completely insensitive to the effective magnetic flux $\phi$, in the low-energy limit. Namely, the AB effect is absent. This rather surprising conclusion follows quite naturally from the present formulation, as follows.

With a gauge transformation, the effect of the flux can be described by the boundary conditions $\varphi_{u}(-L)=$ $\varphi_{l}(-L)$ at the left junction and $\varphi_{u}(L)=\varphi_{l}(L)+\phi$ at the right one. They determine the boundary values of the antisymmetric combination as $\varphi_{a}(-L)=0$ and $\varphi_{a}(L)=\phi$. The current on the ring is just a superposition of the symmetric flow between the two leads described by $\varphi_{s}$ 
and the persistent current described by $\varphi_{a}$. Only the latter depends on the flux $\phi$. As it is only the symmetric combination $\varphi_{s}$ that couples with the leads, the flux $\phi$ has no effect on the transmission between the leads.

If we consider the system with fermions instead of bosons in the same geometry, the $\mathrm{AB}$ effect must be present, at least when the fermions are non-interacting. As the non-interacting fermions in one dimension corresponds to the TL liquid with $g=1$, our result might appear in contradiction. However, there is actually no contradiction, because our analysis applies only to the "strongly coupled" b.c. (6). In the system of noninteracting electrons, the junction is described by a different b.c.. The "strongly coupled" b.c. is expected to be stable even in a fermionic system, when the interaction is sufficiently attractive $(g>9)[11]$. For such a system, the AB effect should be absent as well.

Finally, we discuss the limitations of and possible corrections to the present results. First, the TL liquid description is only valid in the low-energy limit. This requires $T, 2 \pi v / L \ll \mu$, where $L$ is the system size (length of the branches), and the chemical potential $\mu=U \rho^{(0)}$ gives the ultraviolet cutoff. [3] When a packet of extra particles is injected, the variation $\Delta \rho$ of the particle density corresponds to a variation of the local chemical potential $\Delta \mu=U \Delta \rho$. Thus $\Delta \rho \ll \rho^{(0)}$ is required for the TL liquid description to apply. For a larger amplitude, the single-particle physics is expected to show up, instead of the collective nature characterized by the TL liquid.

The "strongly coupled" b.c. (6), which we assumed in the present Letter, is exact only in the low-energy limit, even when the bulk is completely described by the TL liquid. There are corrections due to finite energy scales present in a realistic system. The leading correction to the results based on the b.c. (6) can be related to, at a sufficiently low energy scale, the leading irrelevant operator with scaling dimension $4 g / 3$. It would give, for example, a positive reflection proportional to $\epsilon^{8 g / 3-2}$ on top of the universal negative reflection $-1 / 3$. Here $\epsilon$ denotes the largest among the possible infrared cutoff scales, such as $T, 2 \pi v / L$, and $U \Delta \rho$. When the interaction is weak, it is difficult to satisfy the conditions for the TL liquid theory to be applicable in the bulk. Interestingly, however, the corrections at the junction vanishes quickly because $g$ is large in such a case.
The authors would like to thank I. Affleck, C. Chamon and C.-Y. Hou for useful discussion on related problems, and S. Furukawa for a valuable comment on the absence of the AB effect. A. T. was supported by JSPS. This work was supported in part by 21st Century COE program at Tokyo Institute of Technology 'Nanometer-Scale Quantum Physics' from MEXT of Japan, the NSF grant No. DMR-0132874, AFOSR, and the Harvard-MIT CUA.

[1] H. Ott et al., Phys. Rev. Lett. 87, 230401 (2001); W. Hänsel et al., Nature (London) 413, 498 (2001); J. Fortágh and C. Zimmermann, Rev. Mod. Phys. 79, 235 (2007), and references therein.

[2] D. Sen, J. Phys. A: Math. Gen. 36, 7517 (2003).

[3] M. A. Cazalilla, J. Phys. B: At. Mol. Opt. Phys. 37, S1 (2004).

[4] M. A. Cazalilla, Europhys. Lett. 59, 793 (2002).

[5] M. T. Batchelor et al., J. Phys. A: Math. Gen. 38, 7787 (2005).

[6] M. Jääskeläinen and S. Stenholm, Phys. Rev. A 66, 023608 (2002); ibid. 66, 053605 (2002).

[7] M. Jääskeläinen and S. Stenholm, Phys. Rev. A 68, 033607 (2003).

[8] E. Andersson et al., Phys. Rev. Lett. 88, 100401 (2002).

[9] F. D. M. Haldane, Phys. Rev. Lett. 47, 1840 (1981); Phys. Rev. Lett. 48, 569 (1982).

[10] C. Nayak et al., Phys. Rev. B 59, 15694 (1999).

[11] C. Chamon, M. Oshikawa, and I. Affleck, Phys. Rev. Lett. 91, 206403 (2003); M. Oshikawa, C. Chemon, and I. Affleck, J. Stat. Mech. P02008 (2006).

[12] M. Olshanii, Phys. Rev. Lett. 81, 938 (1998).

[13] E. H. Lieb and W. Liniger, Phys. Rev. 130, 1605 (1963); E. H. Lieb, ibid. 130, 1616 (1963).

[14] I. Affleck et al., J. Stat. Mech. P10003 (2004)

[15] H. Saleur, "Lectures on Non Perturbative Field Theory and Quantum Impurity Problems"; cond-mat/9812110.

[16] D. L. Maslov and M. Stone, Phys. Rev. B 52, R5539 (1995); I. Safi and H. J. Schulz, ibid. 52, R17040 (1995)

[17] I. Safi and H. J. Schulz, "Correlated Fermions and Transport in Mesoscopic Systems", edited by T. Martin, G. Montambaux and J. Tran Thanh Van. Editions Frontieres, Gif-sur-Yvette (1996); cond-mat/9711082.

[18] S. -L. Zhu et al., Phys. Rev. Lett 97, 240401 (2006), and see the references therein.

[19] G. Juzeliunas et al., Phys. Rev. A 71, 053614 (2005). 